\title{
A benchmark of electrostatic methods performance in relative binding free energy calculations
}

\author{
Yunhui Ge, ${ }^{\dagger}$ David F. Hahn, ${ }^{\dagger}$ and David L. Mobley*, \\ †Department of Pharmaceutical Sciences, University of California, Irvine, CA 92697, USA \\ ¥Computational Chemistry, Janssen Research $\&$ Development, Turnhoutseweg 30, Beerse \\ B-2340, Belgium \\ \Departments of Pharmaceutical Sciences and Chemistry, University of California, Irvine, \\ CA 92697, USA \\ E-mail: dmobley@mobleylab.org
}

\begin{abstract}
Relative free energy calculations are fast becoming a critical part of early stage pharmaceutical design, making it important to know how to obtain the best performance with these calculations in applications which could span hundreds of calculations and molecules. In this work, we compared two different treatments of long-range electrostatics, Particle Mesh Ewald (PME) and Reaction Field (RF), in relative binding free energy calculations using a non-equilibrium switching protocol. We found simulations using RF achieve comparable results as those using PME but gain more efficiency when using CPU and similar performance using GPU. The results from this work encourage more use of RF in molecular simulations.
\end{abstract}




\section{INTRODUCTION}

The lead optimization stage is an important part of pharmaceutical drug discovery, involving optimization of several key chemical and biophysical properties in order to ensure candidate compounds have adequate selective binding to their target while also having appropriate other properties to potentially become a new pharmaceutical. Lead optimization efforts always require adequate ligand binding affinity for the target, making this a critical design criterion, and one which is a target for predictive methods. Alchemical relative binding free energy (RBFE) calculations based on molecular dynamics (MD) simulations and statistical mechanics have shown promise in providing reliable predictions to guide experimental work in the context of real drug discovery projects. ${ }^{1-3}$

RBFE calculations compare the potency of two structurally related ligands by transforming one ligand into another via an unphysical or alchemical pathway. This transformation is performed in both the protein-ligand complex and in the solution state to form a closed thermodynamic cycle. The RBFE of the ligands simulated can be calculated through two opposite legs in this cycle. ${ }^{4}$

To calculate free energies, these alchemical transformations can be performed via either an equilibrium or non-equilibrium protocol. In general, a number of intermediate simulations are conducted along an alchemical path between the two physical end states, with these simulations being either equilibrium or nonequilibrium depending on the approach chosen. While the free energy difference of interest depends only on the physical end states in the limit of adequate sampling, these intermediate states serve to help obtain converged results and provide sampling which is hopefully adequate. Equilibrium free energy calculations run an equilibrium simulation at each intermediate state as well as the end states which are physically meaningful. In contrast, the non-equilibrium protocol simulates the end states at equilibrium, potentially spending considerably more time there, and only runs short simulations to switch between end states. However, running a large number of switching trials is critical in this case. It is still under debate which of the two protocols is more 
efficient, with different studies drawing different conclusions, ${ }^{5-8}$ and the choice of protocol is beyond the scope of this paper. This work follows the protocol deployed in a previous work $^{9}$ in which a non-equilibrium approach was used, though our results may generalize to equilibrium approaches as well.

Generally, modern MD engines (e.g., AMBER, GROMACS, CHARMM, etc) support different approaches for simulations, including RBFE calculations. Among these software packages, GROMACS is an open source package which is widely used for molecular simulations and achieves a reasonable level of reproducibility in RBFE calculations. ${ }^{10}$ Moreover, tools (e.g., pmx ${ }^{11,12}$ ) allow easy high-throughput applications of GROMACS RBFE simulations, providing a workflow spanning from initial coordinate files to final free energy estimates. This leads to reproducible and easier conduction of RBFE calculations using GROMACS.

Long-range electrostatics interactions are critical in modeling molecular motions in simulations. Due to the computational complexity of such long-range interactions, it becomes challenging to design accurate and efficient methods to describe such interactions. An excellent review of methods for computing the long-range electrostatics interactions in biomolecular simulations can be found here. ${ }^{13}$

Among a number of existing methods, Particle Mesh Ewald (PME) is perhaps the most broadly used. It is based on the Ewald approach which is a classic method to exactly calculate the electrostatic potential ${ }^{14}$ and is chosen as a starting point for further adjustments for better efficiency. In Ewald summation, the interaction potential is split into a short-range term (direct sum) and a long-range term (reciprocal sum). The direct part converges fast using a fixed cutoff, and the efficiency of the method is determined by the reciprocal part. In PME, the reciprocal sum is computed by solving Poisson's equation for a charge distribution on a mesh via two Fast Fourier transform (FFT) which accelerates the calculation. The PME method finds a balance between the accuracy and efficiency and is widely used in molecular simulations. Additionally, a number of other approaches to long-range electrostatics use similar adaptations of Ewald-based techniques. 
Another popular option to compute electrostatics interactions is Reaction Field (RF). The RF method only computes the interactions up to a cutoff distance and implicitly treats any interactions beyond the cutoff in a mean-field manner using an appropriate dielectric constant. In this way, electrostatics interactions can be calculated efficiently while hopefully maintaining enough accuracy. However for relatively inhomogeneous systems (e.g., membranes), using RF may introduce artifacts and lead to incorrect modeling of the system. For example, lattice sum electrostatics can be used to represent effectively infinite membranes when simulating membrane proteins, but RF requires a continuum treatment of long-range interactions. Still, RF sees considerable use in the field and can be appealing for suitable system geometries.

In this work, we compare the performance of PME and RF methods in RBFE calculations. While the PME method is widely used in RBFE simulations, it is still unclear how the RF method performs in such calculations.

\section{METHODS}

Selected targets. We selected the targets TYK2 ${ }^{15}$ and CDK2 ${ }^{16}$ for benchmark. For the TYK2 system ( 60000 atoms), 24 perturbations were simulated. Simulations of some perturbations crashed due to a combined effect of force field parameters, construction of hybrid topologies and the simulation engine. This issue is beyond the scope of this paper and will be further explored in the future work. For the larger system CDK2 ( 110000

atoms), a smaller set of 6 perturbations was selected to validate the system independence of the former results. Both targets are part of several RBFE benchmark studies. ${ }^{2,17,18}$ A table of successfully simulated perturbations can be found in Table S1, S2, S3, S4, S5.

Molecular Dynamics Simulations. The simulations were performed using GROMACS (2021-dev-20200320-89f1227-unknown) with a patch optimizing PME performance on GPU (https://gerrit.gromacs.org/c/gromacs/+/13382). 
For each perturbation, two sets of simulations were prepared: solvated ligands and ligandprotein complexes. The initial ligand and protein structures were obtained from a previous published work. ${ }^{9}$

The ligand was parameterized using Open Force Field version 1.0.0 (codenamed "Parsley"). ${ }^{19}$ Hybrid structures and topologies for the ligand pairs were generated using pmx following a single topology approach. ${ }^{11,12}$ The workflow established a mapping between atoms of two ligands based on the maximum common substructure and conformational alignment while minimizing perturbation and stabilizing the system.

The AMBER ff99sb*ILDN force field ${ }^{20-22}$ was used for protein parameterization. Dodecahedral boxes were filled with TIP3P explicit solvent model solvated ligand pairs/ligandprotein complexes and counterions $(150 \mathrm{mM} \mathrm{NaCl})$.

For each perturbation, two states were prepared for both in-solution/bound state ligands: state $\mathrm{A}$ and state $\mathrm{B}$, representing ligand 1 and ligand 2, respectively. An energy minimization was first performed, followed by a 10 ps NVT equilibration at $298 \mathrm{~K}$. Then the production equilibrium simulation (in the NPT ensemble) was performed for $6 \mathrm{~ns}$ at $298 \mathrm{~K}$ and a pressure of 1 bar. 80 snapshots were extracted from the production simulation. For each snapshot, a non-equilibrium transformation from state A to B (and vice versa) was performed during 50 ps. For each perturbation, 3 replicas of the series of simulations described above were performed leading to a total of $60 \mathrm{~ns}$ simulation data to calculate the free energy differences for the ligands in their in-solution/bound states.

The stochastic dynamics thermostat was used to control the temperature in the simulations. The Parrinello-Rahman barostat ${ }^{23}$ was applied to keep the pressure constant. All bond lengths were constrained using the LINCS algorithm. ${ }^{24}$ The van der Waals interactions were smoothly switched off between 1.0 and $1.1 \mathrm{~nm}$. A dispersion correction for energy and pressure was used. The non-bonded interactions for the alchemical transitions were treated with a modified soft-core potential. ${ }^{25}$

Two different methods implemented in GROMACS were used to treat long-range electro- 
static interactions: Particle Mesh Ewald (PME) and Reaction Field (RF). For simulations using PME, a direct space cutoff of $1.1 \mathrm{~nm}$ and a Fourier grid spacing of $0.12 \mathrm{~nm}$ were used. For simulations using RF, a dielectric constant $\left(\epsilon_{r f}\right)$ of 78.3 , the dielectric constant of water at $298 \mathrm{~K}$ was used. ${ }^{26}$

Both CPU and GPU simulations were performed using PME or RF electrostatics. In the rest of the paper, we denote CPU-PME, CPU-RF, GPU-PME and GPU-RF to represent the hardware and methods for long-range electrostatic interactions treatment.

\section{RESULTS}

Predicted relative binding free energies from simulations using RF show good agreement with those from PME. The relative binding free energies $(\Delta \Delta G)$ were calculated for a set of modifications of TYK2 which is also included in a standard test set for relative free energy calculations (the set commonly referred to as Schrödinger's "JACS set" 17 from a key paper in JACS on large-scale free energy calculations). ${ }^{2,18}$ Figure 1 summarizes the computed values using RF/PME on CPU/GPU. The uncertainty estimates were performed by 1000 bootstrapping trials and are reported in Figure 1 as $x_{x_{\text {low }}}^{x_{\text {high }}}$ where $x$ is the mean value, $x_{\text {high }}$ and $x_{\text {low }}$ indicate $95 \%$ confidence intervals. The averaged root-mean-square error (RMSE) and mean unsigned error (MUE) of $\Delta \Delta G$ for CPU-PME versus CPU-RF are 0.44 and $0.33 \mathrm{kcal} / \mathrm{mol}$ and for GPU-PME versus GPU-RF are $0.55,0.43 \mathrm{kcal} / \mathrm{mol}$, respectively (Figure 1a-b). A cross-platform (CPU vs GPU) comparison also shows essentially the same level of agreement between results using RF and PME (Figure 1c-d) suggesting the trend observed is independent on the hardware.

Simulations using RF are more efficient than using PME. Given that PME and RF achieve a good level of agreement of calculated $\Delta \Delta G$ values, our focus shifts to computational efficiency, where RF is generally less computationally demanding than using PME. The simulation performance (in the unit of ns/day) was analyzed from the 6-ns equilibrium NPT 

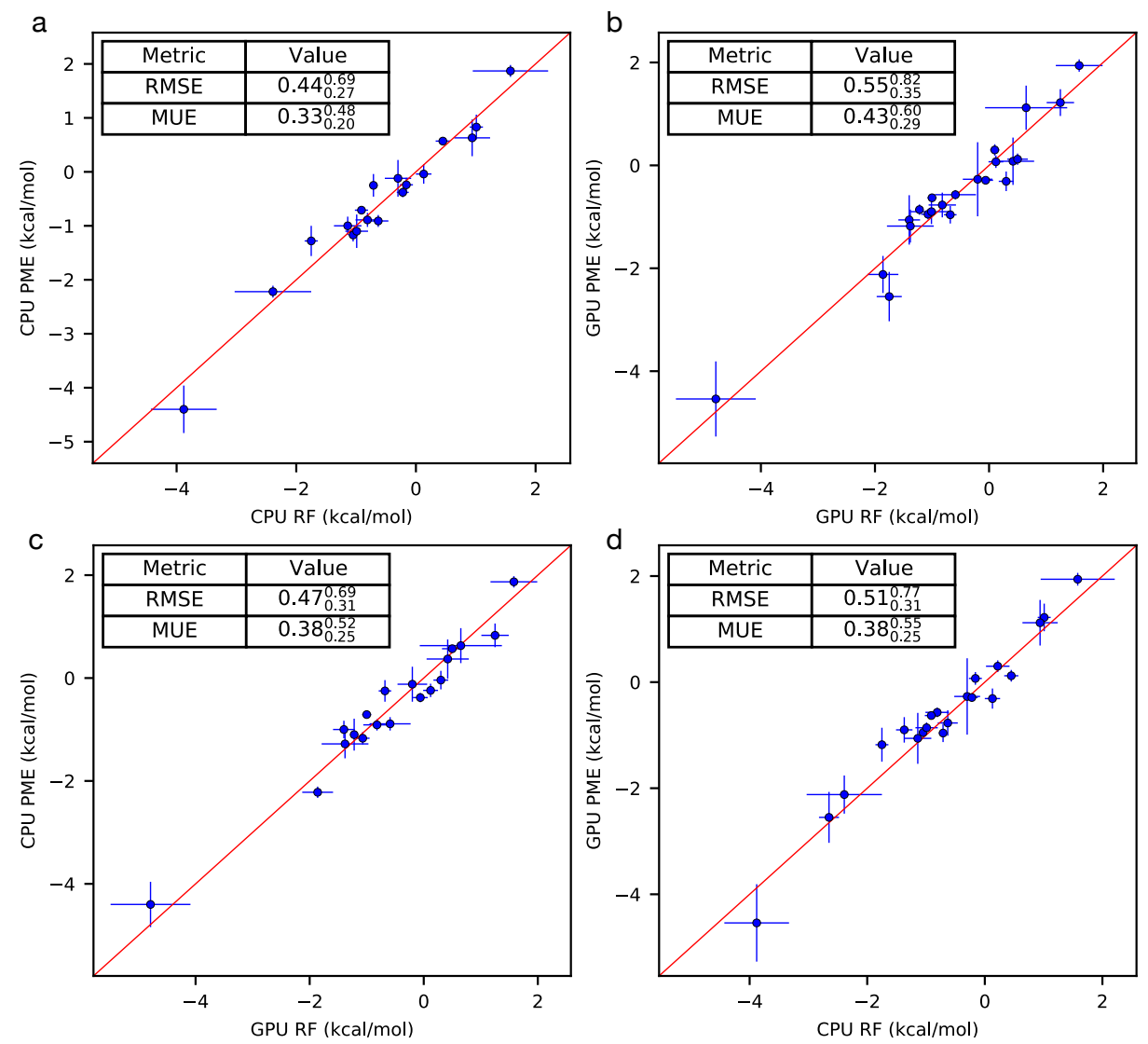

Figure 1: Calculated $\Delta \Delta G$ values of TYK2 ligands using RF/PME on CPU/GPU. Overall, a good agreement is achieved between using PME and RF on CPU/GPU. The uncertainty estimates are calculated by bootstrapping, using 100 bootstrap samples.

simulation and is summarized in Figure 2 where different colors represent using RF/PME on CPU/GPU. The uncertainties were estimated using the standard deviations across different edges. As mentioned in Methods, the simulations were performed in-solution and ligandbound state in which $5908 \pm 221$ atoms and $62290 \pm 6$ atoms were simulated, respectively. For the (less costly) in-solution ligand simulations, using RF on CPU is $\sim 40 \%$ faster than using PME on average (Figure 2a) and is similar to PME when simulated on GPU considering the uncertainties (Figure 2b). For the more costly bound state simulation, a similar trend is observed in CPU simulations (Figure 2c). However, using RF on GPU is $5 \%$ slower than using PME on average (Figure 2d).

The results from Figure 2 show that using RF is faster than PME in most cases for 
the different system sizes and hardware tested here. It is notable that the GROMACS version used in these simulations was specifically optimized for PME performance on GPU (https://gerrit.gromacs.org/c/gromacs/+/13382). Thus, it is not surprising that PME outperformed RF on GPUs (slightly) in our tests here. However, the difference between PME and RF on GPU performance is only minor ( 5\%). Possibly a similar optimization of GROMACS for RF on GPUs could yield substantial performance gains.

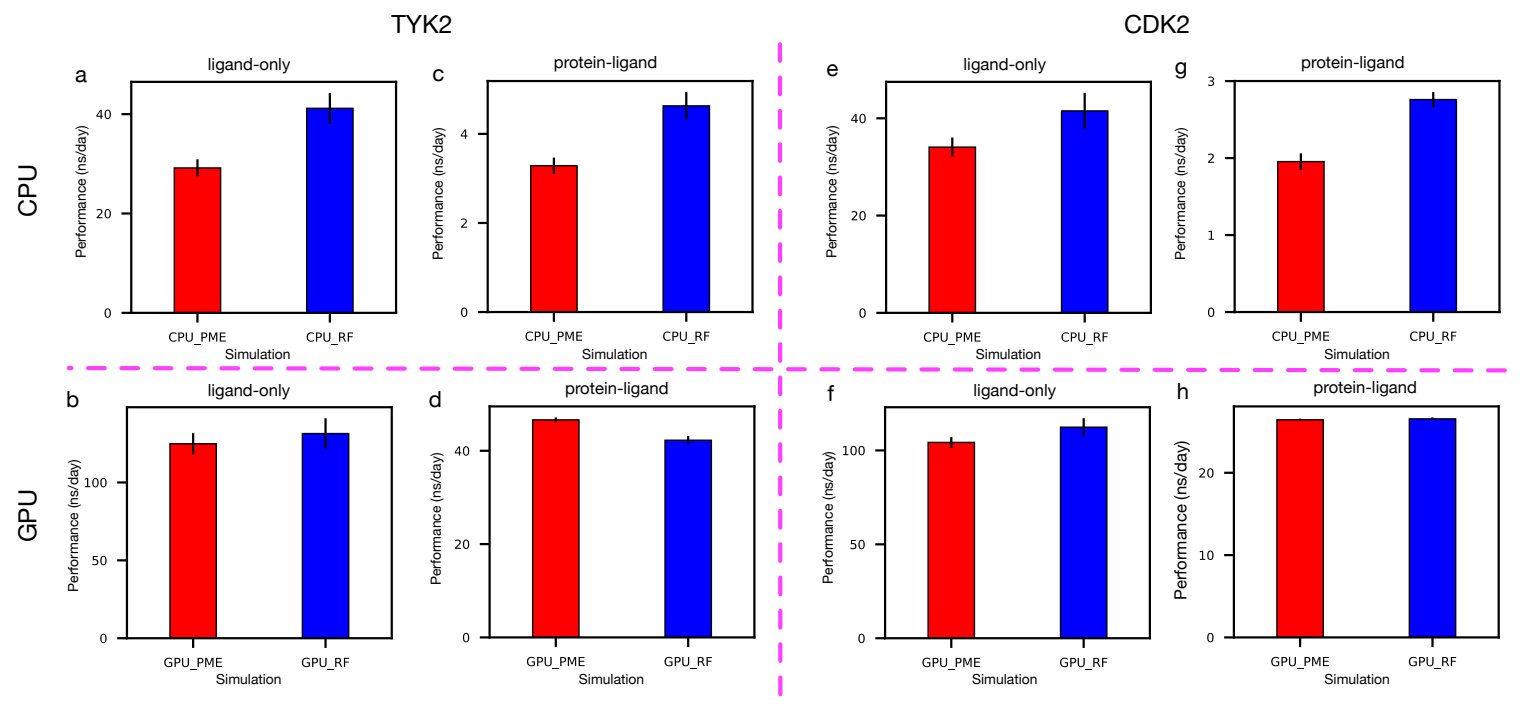

Figure 2: Performance difference between using RF/PME on CPU/GPU in TYK2 simulations of (a-b) ligand-only in-solution, (c-d) protein-ligand complex and in CDK2 simulations of (e-f) ligand-only in-solution, (g-h) protein-ligand complex.

To verify the observed trends in TYK2 simulations, 6 selected edges from CDK2 were simulated using the same protocol described in METHOD. CDK2 is a larger system than TYK2 and has $5602 \pm 373$ and $106908 \pm 5$ atoms in in-solution and ligand-bound state simulation, respectively. Similar to the results of TYK2, simulations using RF are also faster ( 20-40\%) than using PME on CPU (Figure 2e,g). Notably, GPU simulations using RF achieves a similar efficiency to PME (Figure 2f,h) which means even with optimizations, PME still cannot surpass RF in efficiency (Figure 2d).

Figure 2 shows that using RF is more efficient than PME on CPUs and is comparable to PME in GPU simulations. This is remarkable given the fact the GROMACS used in 
this work is optimized for PME performance on GPU. RF could perhaps become even more promising with further optimizations.

On average, the protein-ligand complex simulation of each TYK2 perturbation took 31.1 hours using RF and 43.8 hours using PME on 8 CPUs ( 30\% performance gain using RF), with the protocol used here. With same setup, the ligand-only simulation of TYK2 took 3.5 and 4.9 hours using RF and PME, respectively (also $\sim 30 \%$ performance gain using RF). When using 1 GPU, a calculation time of 3.4 hours using RF and 3.1 hours using PME was needed for protein-ligand complex simulations ( 10\% performance gain using PME). In ligand-only simulation on $1 \mathrm{GPU}$, an average time of 1.1 and 1.2 hours was needed using $\mathrm{RF}$ and PME, respectively ( 10\% performance gain using RF).

Here, while we find that PME and RF achieve comparable results within uncertainty, this is with present-day force fields, and may not always be the case in future studies. It is not too difficult to imagine a scenario where force fields might be optimized for the best performance with a particular model of long range electrostatics, such as a force field designed for use with PME, or one for use with RF. Once force fields are tuned for specific electrostatics treatments, it is reasonable to expect that results might differ in quality if the electrostatics treatment is changed. To some extent, we already see such tuning taking place for water models (e.g. the SPC/E, TIP4P-Ew, and TIP5P-E models designed for use with Ewald-based electrostatics ${ }^{27-31}$ ), though not yet for other components of the force field to our knowledge. Still, results from these water studies indicate that electrostatics treatment, or at least the switch from cutoff based to lattice-sum electrostatics, impacts bulk properties enough that the choice of electrostatics treatment should perhaps be considered part of the force field. So, perhaps future force fields should require a particular choice of long range electrostatics treatment, and separate Ewald-based and RF-based versions should be fitted. 


\section{CONCLUSION}

The treatment of long-range electrostatic interactions is critical for a correct modeling of (bio)molecular systems in molecular dynamics simulations. Due to the long-range nature and $\mathrm{N}^{2}$-scaling of electrostatic interactions, they are computationally the most demanding terms in the force field evaluation. Both PME and RF are popular methods of treating long range electrostatic interactions. PME is widely used and attempts to find a good balance between efficiency and accuracy, but we find that RF achieves higher efficiency, although it may have some limitations in relatively inhomogeneous systems such as for membrane simulations.

This work is focused on comparing the results and performance of PME and RF for relative binding free energy calculations. We found the calculated $\Delta \Delta G$ values are in good agreement using PME and RF whereas simulations using RF are comparable or faster than PME on both CPU and GPU. Taken the results presented here, we suggest that RF may be a promising option for relative free energy simulations because, at least in GROMACS, it is less computationally demanding while retaining comparable accuracy to PME. This advantage may be particularly helpful in cases where a large number of simulations are needed (e.g., in the lead optimization stage of the drug discovery process). Thus we recommend free energy calculations with RF be considered as a viable option, at least for homogeneous systems.

\section{ACKNOWLEDGEMENTS}

DLM appreciates financial support from the National Institutes of Health (R01GM108889 and R01GM132386). We appreciate the Open Force Field Consortium for its support of the Open Force Field Initiative, which provided software infrastructure used in this work. 


\section{Notes}

All input files for simulations, data and scripts for analysis are freely available at https: //github.com/MobleyLab/PME-RF-benchmark.

\section{References}

(1) Cournia, Z.; Allen, B.; Sherman, W. Relative Binding Free Energy Calculations in Drug Discovery: Recent Advances and Practical Considerations. Journal of Chemical Information and Modeling 2017, 57, 2911-2937.

(2) Song, L. F.; Lee, T.-S.; Zhu, C.; York, D. M.; Merz, K. M. Using AMBER18 for Relative Free Energy Calculations. Journal of Chemical Information and Modeling 2019, 59, $3128-3135$.

(3) Schindler, C. E. M. et al. Large-Scale Assessment of Binding Free Energy Calculations in Active Drug Discovery Projects. Journal of Chemical Information and Modeling 2020, 60, 5457-5474.

(4) Mey, A. S. J. S.; Allen, B.; Macdonald, H. E. B.; Chodera, J. D.; Kuhn, M.; Michel, J.; Mobley, D. L.; Naden, L. N.; Prasad, S.; Rizzi, A.; Scheen, J.; Shirts, M. R.; Tresadern, G.; Xu, H. Best Practices for Alchemical Free Energy Calculations. arXiv 2020,

(5) Ytreberg, F. M.; Swendsen, R. H.; Zuckerman, D. M. Comparison of Free Energy Methods for Molecular Systems. The Journal of Chemical Physics 2006, 125, 184114.

(6) Goette, M.; Grubmüller, H. Accuracy and Convergence of Free Energy Differences Calculated from Nonequilibrium Switching Processes. Journal of Computational Chemistry 2009, 30, 447-456.

(7) Yildirim, A.; Wassenaar, T. A.; van der Spoel, D. Statistical Efficiency of Methods for 
Computing Free Energy of Hydration. The Journal of Chemical Physics 2018, 149, 144111.

(8) Baumann, H.; Gapsys, V.; de Groot, B. L.; Mobley, D. Challenges Encountered Applying Equilibrium and Non-Equilibrium Binding Free Energy Calculations. ChemRxiv 2020,

(9) Gapsys, V.; Pérez-Benito, L.; Aldeghi, M.; Seeliger, D.; van Vlijmen, H.; Tresadern, G.; de Groot, B. L. Large Scale Relative Protein Ligand Binding Affinities Using NonEquilibrium Alchemy. Chemical Science 2020, 11, 1140-1152.

(10) Loeffler, H. H.; Bosisio, S.; Duarte Ramos Matos, G.; Suh, D.; Roux, B.; Mobley, D. L.; Michel, J. Reproducibility of Free Energy Calculations across Different Molecular Simulation Software Packages. Journal of Chemical Theory and Computation 2018, 14, $5567-5582$.

(11) Seeliger, D.; de Groot, B. L. Protein Thermostability Calculations Using Alchemical Free Energy Simulations. Biophysical Journal 2010, 98, 2309-2316.

(12) Gapsys, V.; Michielssens, S.; Seeliger, D.; de Groot, B. L. Pmx: Automated Protein Structure and Topology Generation for Alchemical Perturbations. Journal of Computational Chemistry 2015, 36, 348-354.

(13) Cisneros, G. A.; Karttunen, M.; Ren, P.; Sagui, C. Classical Electrostatics for Biomolecular Simulations. Chemical Reviews 2014, 114, 779-814.

(14) Ewald, P. P. Die Berechnung optischer und elektrostatischer Gitterpotentiale. Annalen der Physik 1921, 369, 253-287.

(15) Liang, J. et al. Lead identification of novel and selective TYK2 inhibitors. European Journal of Medicinal Chemistry 2013, 67, 175-187. 
(16) Hardcastle, I. R. et al. N2-substituted O6-cyclohexylmethylguanine derivatives: Potent inhibitors of cyclin-dependent kinases 1 and 2. Journal of Medicinal Chemistry 2004, $47,3710-3722$.

(17) Wang, L. et al. Accurate and Reliable Prediction of Relative Ligand Binding Potency in Prospective Drug Discovery by Way of a Modern Free-Energy Calculation Protocol and Force Field. Journal of the American Chemical Society 2015, 137, 2695-2703.

(18) Kuhn, M.; Firth-Clark, S.; Tosco, P.; Mey, A. S. J. S.; Mackey, M.; Michel, J. Assessment of Binding Affinity via Alchemical Free-Energy Calculations. Journal of Chemical Information and Modeling 2020, 60, 3120-3130.

(19) Qiu, Y. et al. Development and Benchmarking of Open Force Field v1.0.0, the Parsley Small Molecule Force Field. ChemRxiv 2020,

(20) Hornak, V.; Abel, R.; Okur, A.; Strockbine, B.; Roitberg, A.; Simmerling, C. Comparison of Multiple Amber Force Fields and Development of Improved Protein Backbone Parameters. Proteins: Structure, Function, and Bioinformatics 2006, 65, 712-725.

(21) Best, R. B.; Hummer, G. Optimized Molecular Dynamics Force Fields Applied to the HelixCoil Transition of Polypeptides. The Journal of Physical Chemistry B 2009, 113, 9004-9015.

(22) Lindorff-Larsen, K.; Piana, S.; Palmo, K.; Maragakis, P.; Klepeis, J. L.; Dror, R. O.; Shaw, D. E. Improved Side-Chain Torsion Potentials for the Amber ff99SB Protein Force Field: Improved Protein Side-Chain Potentials. Proteins: Structure, Function, and Bioinformatics 2010, 78, 1950-1958.

(23) Parrinello, M.; Rahman, A. Polymorphic Transitions in Single Crystals: A New Molecular Dynamics Method. Journal of Applied Physics 1981, 52, 7182-7190. 
(24) Hess, B.; Bekker, H.; Berendsen, H. J. C. LINCS: A Linear Constraint Solver for Molecular Simulations. Journal of Computational Chemistry 1997, 18, 1463-1472.

(25) Gapsys, V.; Seeliger, D.; de Groot, B. L. New Soft-Core Potential Function for Molecular Dynamics Based Alchemical Free Energy Calculations. Journal of Chemical Theory and Computation 2012, 8, 2373-2382.

(26) Malmberg, C. G.; Maryott, A. A. Dielectric Constant of Water From 0-Degrees-C to 100-Degrees-C. Journal of Research of the National Bureau of Standards 1956, 56, 1-8.

(27) Berendsen, H. J. C.; Grigera, J. R.; Straatsma, T. P. The Missing Term in Effective Pair Potentials. The Journal of Physical Chemistry 1987, 91, 6269-6271.

(28) Chatterjee, S.; Debenedetti, P. G.; Stillinger, F. H.; Lynden-Bell, R. M. A Computational Investigation of Thermodynamics, Structure, Dynamics and Solvation Behavior in Modified Water Models. The Journal of Chemical Physics 2008, 128, 124511.

(29) Horn, H. W.; Swope, W. C.; Pitera, J. W.; Madura, J. D.; Dick, T. J.; Hura, G. L.; Head-Gordon, T. Development of an Improved Four-Site Water Model for Biomolecular Simulations: TIP4P-Ew. The Journal of Chemical Physics 2004, 120, 9665-9678.

(30) Horn, H. W.; Swope, W. C.; Pitera, J. W. Characterization of the TIP4P-Ew Water Model: Vapor Pressure and Boiling Point. The Journal of Chemical Physics 2005, 123, 194504.

(31) Rick, S. W. A Reoptimization of the Five-Site Water Potential (TIP5P) for Use with Ewald Sums. The Journal of Chemical Physics 2004, 120, 6085-6093.

\section{Supplementary Information}

\section{Supporting Tables}


Table S1: Simulated perturbations of TYK2 (CPU-PME) and the number of particles.

\begin{tabular}{|c|c|c|c|}
\hline Ligand1 & Ligand 2 & No. atoms (ligand-only) & No. atoms (protein-ligand) \\
\hline ejm-44 & ejm-55 & 5954 & 62290 \\
ejm-49 & ejm-31 & 6521 & 62288 \\
ejm-31 & ejm-46 & 5888 & 62281 \\
jmc-28 & jmc-27 & 5886 & 62282 \\
ejm-42 & ejm-48 & 6014 & 62290 \\
ejm-31 & ejm-43 & 5950 & 62286 \\
ejm-50 & ejm-42 & 5630 & 62272 \\
ejm-42 & ejm-55 & 5633 & 62275 \\
jmc-23 & ejm-46 & 5884 & 62277 \\
ejm-31 & ejm-45 & 5638 & 62283 \\
ejm-55 & ejm-54 & 5729 & 62278 \\
ejm-45 & ejm-42 & 5639 & 62284 \\
ejm-31 & ejm-48 & 5960 & 62284 \\
ejm-47 & ejm-31 & 5906 & 62290 \\
ejm-47 & ejm-55 & 5915 & 62290 \\
ejm-44 & ejm-42 & 5956 & 62295 \\
jmc-23 & jmc-30 & 5884 & 62277 \\
jmc-28 & jmc-30 & 5887 & 62286 \\
ejm-49 & ejm-50 & 6517 & 62290 \\
\hline
\end{tabular}


Table S2: Simulated perturbations of TYK2 (CPU-RF) and the number of particles.

\begin{tabular}{|c|c|c|c|}
\hline Ligand1 & Ligand 2 & No. atoms (ligand-only) & No. atoms (protein-ligand) \\
\hline ejm-44 & ejm-55 & 5954 & 62290 \\
ejm-49 & ejm-31 & 6521 & 62288 \\
ejm-31 & ejm-46 & 5888 & 62281 \\
jmc-28 & jmc-27 & 5886 & 62282 \\
ejm-42 & ejm-48 & 6014 & 62290 \\
ejm-31 & ejm-43 & 5950 & 62286 \\
ejm-50 & ejm-42 & 5630 & 62272 \\
ejm-42 & ejm-55 & 5633 & 62275 \\
jmc-23 & ejm-46 & 5884 & 62277 \\
ejm-55 & ejm-54 & 5729 & 62278 \\
ejm-45 & ejm-42 & 5639 & 62284 \\
ejm-31 & ejm-48 & 5960 & 62284 \\
ejm-47 & ejm-31 & 5906 & 62290 \\
ejm-47 & ejm-55 & 5915 & 62290 \\
ejm-44 & ejm-42 & 5956 & 62295 \\
jmc-23 & jmc-27 & 5886 & 62276 \\
ejm-43 & ejm-55 & 5951 & 62281 \\
jmc-23 & jmc-30 & 5884 & 62277 \\
jmc-28 & jmc-30 & 5887 & 62286 \\
ejm-42 & ejm-54 & 5640 & 62282 \\
ejm-49 & ejm-50 & 6517 & 62290 \\
\hline
\end{tabular}


Table S3: Simulated perturbations of TYK2 (GPU-PME) and the number of particles.

\begin{tabular}{|c|c|c|c|}
\hline Ligand1 & Ligand 2 & No. atoms (ligand-only) & No. atoms (protein-ligand) \\
\hline ejm-44 & ejm-55 & 5954 & 62290 \\
ejm-49 & ejm-31 & 6521 & 62288 \\
ejm-31 & ejm-46 & 5888 & 62281 \\
jmc-28 & jmc-27 & 5886 & 62282 \\
ejm-42 & ejm-48 & 6014 & 62290 \\
ejm-31 & ejm-43 & 5950 & 62286 \\
ejm-50 & ejm-42 & 5630 & 62272 \\
ejm-42 & ejm-55 & 5633 & 62275 \\
jmc-23 & ejm-46 & 5884 & 62277 \\
ejm-31 & ejm-45 & 5638 & 62283 \\
ejm-55 & ejm-54 & 5729 & 62278 \\
ejm-45 & ejm-42 & 5639 & 62284 \\
ejm-31 & ejm-48 & 5960 & 62284 \\
ejm-47 & ejm-31 & 5906 & 62290 \\
ejm-47 & ejm-55 & 5915 & 62290 \\
ejm-44 & ejm-42 & 5956 & 62295 \\
jmc-23 & jmc-27 & 5886 & 62276 \\
ejm-43 & ejm-55 & 5951 & 62281 \\
jmc-23 & jmc-30 & 5884 & 62277 \\
jmc-28 & jmc-30 & 5887 & 62286 \\
ejm-42 & ejm-54 & 5640 & 62282 \\
ejm-49 & ejm-50 & 6517 & 62290 \\
\hline
\end{tabular}


Table S4: Simulated perturbations of TYK2 (GPU-RF) and the number of particles.

\begin{tabular}{|c|c|c|c|}
\hline Ligand1 & Ligand 2 & No. atoms (ligand-only) & No. atoms (protein-ligand) \\
\hline ejm-44 & ejm-55 & 5954 & 62290 \\
ejm-49 & ejm-31 & 6521 & 62288 \\
ejm-31 & ejm-46 & 5888 & 62281 \\
jmc-28 & jmc-27 & 5886 & 62282 \\
ejm-42 & ejm-48 & 6014 & 62290 \\
ejm-31 & ejm-43 & 5950 & 62286 \\
ejm-50 & ejm-42 & 5630 & 62272 \\
ejm-42 & ejm-55 & 5633 & 62275 \\
jmc-23 & ejm-46 & 5884 & 62277 \\
ejm-31 & ejm-45 & 5638 & 62283 \\
ejm-55 & ejm-54 & 5729 & 62278 \\
ejm-45 & ejm-42 & 5639 & 62284 \\
ejm-31 & ejm-48 & 5960 & 62284 \\
ejm-47 & ejm-31 & 5906 & 62290 \\
ejm-47 & ejm-55 & 5915 & 62290 \\
ejm-44 & ejm-42 & 5956 & 62295 \\
jmc-23 & jmc-27 & 5886 & 62276 \\
ejm-43 & ejm-55 & 5951 & 62281 \\
jmc-23 & jmc-30 & 5884 & 62277 \\
jmc-28 & jmc-30 & 5887 & 62286 \\
ejm-42 & ejm-54 & 5640 & 62282 \\
ejm-49 & ejm-50 & 6517 & 62290 \\
\hline
\end{tabular}

Table S5: Simulated perturbations of CDK2 and the number of particles.

\begin{tabular}{|c|c|c|c|}
\hline Ligand1 & Ligand 2 & No. atoms (ligand-only) & No. atoms (protein-ligand) \\
\hline 22 & 1h1r & 4978 & 106892 \\
1oiu & 26 & 5885 & 106905 \\
26 & 1h1q & 5762 & 106902 \\
17 & $1 \mathrm{~h} 1 \mathrm{q}$ & 4987 & 106904 \\
1oiy & 1oi9 & 5675 & 106908 \\
17 & 21 & 4984 & 106895 \\
\hline
\end{tabular}




\section{Graphical TOC Entry}

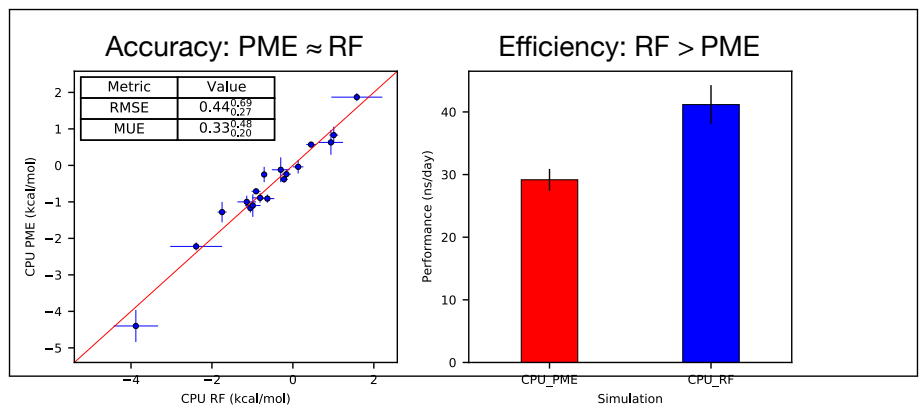

